\title{
Effectiveness of Clinical, Surgical and Percutaneous Treatment to Prevent Cardiovascular Events in Patients Referred for Elective Coronary Angiography: An Observational Study
}

This article was published in the following Dove Press journal:

Vascular Health and Risk Management

\author{
Adriana Silveira Almeida (1D \\ Sandra C Fuchs (D) $^{1,2}$ \\ Felipe C Fuchs $\mathbb{D}^{1,2}$ \\ Aline Gonçalves Silva (D) \\ Marcelo Balbinot Lucca (D) \\ Samuel Scopel (D) \\ Flávio D Fuchs $\mathbb{D}^{1,2}$ \\ 'Postgraduate Studies Program in \\ Cardiology, School of Medicine, \\ Universidade Federal do Rio Grande do \\ Sul, Porto Alegre, RS, Brazil; ${ }^{2}$ Division of \\ Cardiology, Hospital de Clinicas de Porto \\ Alegre, Universidade Federal do Rio \\ Grande do Sul, Porto Alegre, RS, Brazil
}

Correspondence: Adriana Silveira Almeida,Av Alegrete,423 Ap I40I, Porto Alegre, RS CEP 90460-100, Brazil $\mathrm{Tel}+5551996510255$

Email adrianasdealmeida@gmail.com
Purpose: To ascertain the most appropriate treatment for chronic, stable, coronary artery disease (CAD) in patients submitted to elective coronary angiography.

Patients and Methods: A total of 814 patients included in the prospective cohort study were referred for elective coronary angiography and were followed up on average for $6 \pm 1.9$ years. Main outcomes were all-cause death, cardiovascular death, non-fatal myocardial infarction (MI) and stroke and late revascularization and their combinations as major adverse cardiac and cerebral events (MACCE): MACCE-1 included cardiovascular death, nonfatal MI, and stroke; MACCE-2 was MACCE-1 plus late revascularization. Survival curves and adjusted Cox proportional hazard models were used to explore the association between the type of treatment and outcomes.

Results: All-cause death was lower in participants submitted to percutaneous coronary intervention $(\mathrm{PCI})(0.41,0.16-1.03, \mathrm{P}=0.057)$ compared to medical treatment (MT). Coronary-artery bypass grafting $(\mathrm{CABG})$ had an overall trend for poorer outcomes: cardiovascular death $2.53(0.42-15.10)$, combined cardiovascular death, nonfatal MI, and stroke $2.15(0.73-6.31)$ and these events plus late revascularization $(2.17,0.86-5.49)$. The corresponding numbers for PCI were $0.27(0.05-1.43)$ for cardiovascular death, $0.77(0.32-1.84)$ for combined cardiovascular death, nonfatal MI, and stroke and 2.35 (1.16-4.77) with the addition of late revascularization. These trends were not influenced by baseline blood pressure, left ventricular ejection fraction and previous MI. Patients with diabetes mellitus had a significantly higher risk of recurrent revascularization when submitted to PCI than CABG.

Conclusion: Patients with confirmed CAD in elective coronary angiography do not have a better prognosis when submitted to $\mathrm{CABG}$ comparatively to medical treatment. Patients treated with PCI had a trend for the lower incidence of combined cardiovascular events, at the expense of additional revascularization procedures. Patients without significant CAD had a similar prognosis than CAD patients treated with medical therapy.

Keywords: stable coronary artery disease, SYNTAX score, coronary artery bypass grafting, percutaneous coronary intervention, myocardial revascularization

\section{Introduction}

Evidence from clinical trials support the choice of treatment for primary prevention of coronary artery disease (CAD) and for the management of acute coronary syndromes. The most appropriate treatment for patients with chronic CAD, 
however, is still uncertain. There is a consensus for the indication of myocardial revascularization therapies, either surgical or percutaneous, in patients with limiting symptoms despite of drug treatment. ${ }^{1}$ It remains to be determined the best treatment for patients with chronic, stable, CAD.

Several randomized clinical trials have compared different strategies of management of chronic CAD. In most, however, the comparison was between coronary artery bypass grafting (CABG) and percutaneous interventions (PCI), without an arm of patients treated medically. Overall, in old trials, there was no significant difference in the mortality rate and incidence of myocardial infarction in patients treated by $\mathrm{CABG}$ or $\mathrm{PCI} .^{2-10}$ In contemporary trials, with modern stents and enhanced surgical technics, there was an advantage of CABG over PCI in patients with complex multivessel disease in the Syntax trial. ${ }^{11}$ In a meta-analysis of six randomized trials with patients with multivessel disease, ${ }^{12}$ CABG was superior to PCI in the prevention of long-term mortality, myocardial infarction and repeat revascularization. In patients with the left main disease, CABG was superior to PCI in the NOBLE trial. ${ }^{13}$

Fewer randomized clinical trials investigated the better management of chronic stable coronary artery disease, including medical treatment as an option. ${ }^{14-16} \mathrm{CABG}$ was superior to medical therapy and PCI in the prevention of myocardial infarction and combined cardiovascular events in the MASS II trial. ${ }^{14}$ In the COURAGE trial, ${ }^{15}$ PCI was not superior to optimal medical therapy alone in reducing the risk of all-cause death and nonfatal myocardial infarction, independently of the presence of ischemia.${ }^{17}$ In the BARI-2D, ${ }^{16}$ restricted to patients with diabetes, the rates of death and major cardiovascular events were similar in patients submitted to revascularization by CABG or PCI compared to patients managed medically. The absence of superiority of therapies of revascularization over medical treatment was demonstrated in a metaanalysis of patients with chronic CAD and a positive test for ischemia. ${ }^{18}$

The performance of medical therapy and strategies of revascularization was investigated in cohort studies as well, many with data from registries. These real-world studies have the advantage of studying all comers and the effectiveness of therapies delivered by professionals with variable training skills. The possibility of indication bias cannot be fully excluded, however. Only old studies compared surgery with medical treatment. ${ }^{10-22}$ These studies are not anymore valid to identify the best strategy, in the face of the dramatic evolution of medical and surgical therapies. Contemporary studies compared CABG with PCI. In the New York registry, ${ }^{23}$ CABG was associated with lower mortality rates, myocardial infarction and repeat revascularization than treatment with drug-eluting stents. In a metaanalysis of observational cohorts comparing CABG versus drug-eluting stents (DES)-PCI, including a total of 24,268 patients with multivessel coronary disease, major adverse cardiac and cerebrovascular events were similar between the two strategies, but there was an excess of redo revascularization after DES-PCI compared to CABG. ${ }^{24}$

The evidences herein revised demonstrate that it is still necessary to investigate the better strategy to deal with chronic CAD. In the face of the large variability of criteria for inclusion in clinical trials and in observational studies, it is worth expanding the investigation to different clinical scenarios. Within this context, this cohort compared the effectiveness of medical treatment alone, PCI and CABG in preventing death and major adverse cardiac or cerebral events (MACCE) in patients with chronic CAD referred for diagnostic angiography.

\section{Patients and Methods}

Study design: cohort study.

\section{Population}

Patients with suspected CAD referred for elective diagnostic coronary angiography in a reference tertiary university-affiliated hospital from November 2006 to July 2014 were sequentially enrolled in the study. Our Division uses to perform around 3000 percutaneous diagnostic and therapeutic procedures and about 600 cardiac surgeries per year. Patients were referred by cardiologists and clinicians from the public health system and private practices. Data for this analysis were prospectively collected and included the clinical indication of the cardiologist and a summary clinical evaluation done at the cath lab. All patients with 40 years of age or over were enrolled. Patients with acute coronary syndromes, valvular heart disease, aortic diseases, previous coronary revascularization, class III or IV heart failure, chronic renal disease (previous medical diagnosis or serum creatinine greater than $1.5 \mathrm{mg} / \mathrm{dL}$ ), history of cancer, or severe psychiatric illness were excluded.

\section{Baseline Evaluation}

The baseline interviews were performed immediately before the coronary angiography, pertaining to demographic information, lifestyle characteristics, education and past medical 
history, using a standardized questionnaire. Demographic data were gathered by self-report skin color, education by self-report years of schooling, and age was calculated subtracting birth from the interview date.

Weight was measured (with the subject in light clothing and barefoot) to the nearest $100 \mathrm{~g}$ with a scale (Plenna ${ }^{\circledR}$, model TINN 00088 Plenna - S.A., São Paulo, Brazil), and height was measured maintaining the Frankfort plane, to the nearest $1 \mathrm{~cm}$. Body mass index was calculated with the average of three measurements.

Three blood pressure measurements were performed using a validated automatic device (OMRON-CP705, Dupont, France). Hypertension was defined as the average of the last two among three blood pressure measurements greater than $140 / 90 \mathrm{mmHg}$, or the use of blood pressurelowering medication.

Blood samples were collected before coronary angiography to measure the lipid profile, high-sensitivity C-reactive protein, creatinine, and glucose. Diabetes mellitus was defined as a patient's self-report of a physician's diagnosis or use of hypoglycemic agents or insulin. Subjects who reported having smoked at least 100 cigarettes during their lifetime were identified as former smokers. Current smokers were those who reported smoking every day or some days at the time of the interview.

The SYNTAX Score (SXscore) was calculated to evaluate the extent and severity of coronary disease, with higher scores indicating more severe disease. Angiographic visual analysis was independently done by two interventional cardiologists, blinded for clinical variables, for the assessment of the SXscore, trained using the SXscore tutorial. In case of disagreement, a third interventionist was consulted and the final decision was reached by consensus. For each patient, the score was calculated prospectively by including all coronary lesions producing $\mathrm{a} \geq 50 \%$ diameter stenosis in vessels $\geq 1.5 \mathrm{~mm}$ in diameter, using the SXscore calculator. Subsequently, they were categorized as high $(>32)$, intermediate $(23-32)$, low SXscore $(<23)$ and no significant CAD (reference category). ${ }^{25}$

Assessment of ten-year risk for a first hard atherosclerotic cardiovascular disease (ASCVD) event, defined as the first occurrence of nonfatal myocardial infarction or coronary heart disease (CHD) death, or fatal or nonfatal stroke, was calculated according to the Guideline on the Assessment of Cardiovascular Risk using a calculator available at http:// www.cardiosource.org/scienceand-quality/practice-guide lines-and-quality-standards/2013-prevention-guidelinetools.aspx.

\section{Criteria for Treatment Allocation}

The interventional cardiology staff assessed the coronary angiograms. Patients without coronary artery stenosis above $50 \%$ of the vessel diameter were not considered for surgical or percutaneous treatment. The attending physicians received the interpretation of the angiograms and were consulted regarding the therapeutic decision when significant CAD was present. Severe disease with multivessel involvement was usually discussed by the heart team, composed by the attending physician, the interventional cardiologist and the cardiovascular surgeon. The final allocation of these patients to the therapeutic alternatives was done according to the heart team's decision or let to the discretion of attending physician. Scores of coronary lesion severity, including the Syntax score, were not routinely used in the clinical scenario.

\section{Outcome Definitions}

The clinical endpoints were all-cause and cardiovascular death, myocardial infarction, stroke, heart failure, late revascularization and combinations of these outcomes as major adverse cardiac and cerebral events: MACCE-1 was defined by cardiovascular death, nonfatal myocardial infarction, and stroke; MACCE-2 was MACCE-1 plus late revascularization.

Deaths were classified according to the ARIC (Atherosclerosis Risk in Communities Study) protocol. ${ }^{26}$ Myocardial infarction and revascularization followed by death in the same hospitalization were adjudicated as cardiovascular death. Sudden death was defined as cardiovascular death, unless obvious non-cardiac causes could be identified.

Heart failure (HF) was defined by history and medical records. Cases of incident heart failure were those that required hospitalization.

Myocardial infarction (MI) was diagnosed by an increase of cardiac biomarkers, in the presence of symptoms or electrocardiogram (ECG) abnormalities suggestive of ischemia. ${ }^{26}$ Patients that were treated for MI in other hospitals had the diagnosis defined on the basis of the discharge report. Stroke was diagnosed by computed tomography (CT) scanning and compatible clinical findings, besides a review of medical records. Late revascularization was considered either by PCI or CABG. Percutaneous and surgical revascularizations based on diagnostic angiography findings, performed until 3 months after the angiography, were defined as index procedures and not considered outcomes. Interventions performed during follow-up, non-directly related to the diagnostic angiography, 
were defined as late revascularizations and included in the MACCE-2 outcome.

\section{Follow-Up Participants}

The follow-up of patients combined different strategies: telephone interviews, registered letters, medical records, death certificates, and interviews with next-of-keen in some cases.

Death certificates were obtained at the State Health Department or hospital records. Verbal autopsies with next-of keen were done, including information on hospitalizations, physician visits, coronary artery disease symptoms, other diseases and treatments, circumstances surrounding the death, and the use of emergency medical services.

All data were evaluated by at least two authors independently, with control of quality on data entry to verify amplitude and consistency. For quality control of the team's performance, $20 \%$ of the protocols were randomly selected to be reviewed by the main investigator.

The methodology of this study was based on the STROBE guidelines (Strengthening the Reporting of Observational Studies in Epidemiology).

\section{Statistical Analysis}

The sample size calculation was performed in the PEPI program (Programs for Epidemiologists) version 4.0. The estimate was based on studies by Hueb et al, ${ }^{14}$ maintaining statistical power of $80 \%$ and a significance of $5 \%$, with a minimum difference in the incidence of the composite endpoint consisting of death or cardiovascular death, myocardial infarction and refractory angina with revascularization of $12 \%$ among the treatment groups. Thus, a minimum of 366 subjects with CAD was estimated, with an additional $20 \%$ to account for potential losses (total of 440 participants).

Quantitative variables were described by mean and standard deviation, in cases of symmetrical distribution, or median and interquartile range, in case of asymmetric distribution, and qualitative parameters through absolute and relative frequencies. Both groups were compared using Analysis of Variance (ANOVA) and the Scheffé test (symmetrical distribution), or the Kruskal-Wallis test followed by the Dunn's test (asymmetrical distribution), in case of quantitative variables, and the Pearson's chisquared test or the Fisher's exact test for qualitative variables (rates and proportions).

Crude and adjusted incidence of all-cause mortality, cardiovascular mortality, MI, stroke, revascularization,
MACCE-1 and MACCE-2 according to the treatment group were computed.

The association between treatments and outcomes was explored in Cox proportional hazard models and described by Kaplan-Meier survival curves.

Unadjusted survival curves and models adjusting for confounding variables were computed. Model 1 included sociodemographic variables; model 2 included the variables from model 1 plus clinical variables; model 3 added the angiographic evaluation (SXscore). Models were run in stratified analyses for clinical baseline conditions: MI, diabetes mellitus (DM) and categories of systolic blood pressure (SBP). The variables included in the models were those traditionally associated with the incidence of cardiovascular outcomes and those that achieved $\mathrm{P}$ value greater or equal 0.2 in the crude analysis. The level of significance was 5\% and data were analyzed with SPSS (Statistical Package for the Social Sciences) version 21.0.

\section{Ethical Aspects}

The study protocol was approved by the Ethics Committee of the Hospital de Clínicas de Porto Alegre, registered under no. 13-0171, which is accredited by the Office for Human Research Protections as an Institutional Review Board. This study was conducted in accordance with the Declaration of Helsinki. All participants provided informed consent.

\section{Results}

\section{Baseline Characteristics and Angiographic Data}

The study flowchart is presented in Figure 1. From 1028 patients electively submitted to diagnostic coronary angiography, 814 were included in the cohort and were followed up on average for $6 \pm 1.9$ years (median 5.7 years). Vital status follow-up was $100 \%$. For non-fatal outcomes, 93.4\% of patients had detailed information collected.

Table 1 shows the baseline clinical and angiographic characteristics according to therapeutic strategy and the cohort without CAD. As expected, patients with CAD had a higher prevalence of risk factors than patients without CAD, for example, diabetes mellitus, hypertension and smoking. The 10-year ASCVD risk was also higher in patients with $\mathrm{CAD}$ and heart failure more prevalent. The complaints of chest pain and dyspnea, however, were more frequent among participants without CAD.

Among patients with CAD, those submitted to surgical revascularization presented a higher prevalence of heart 


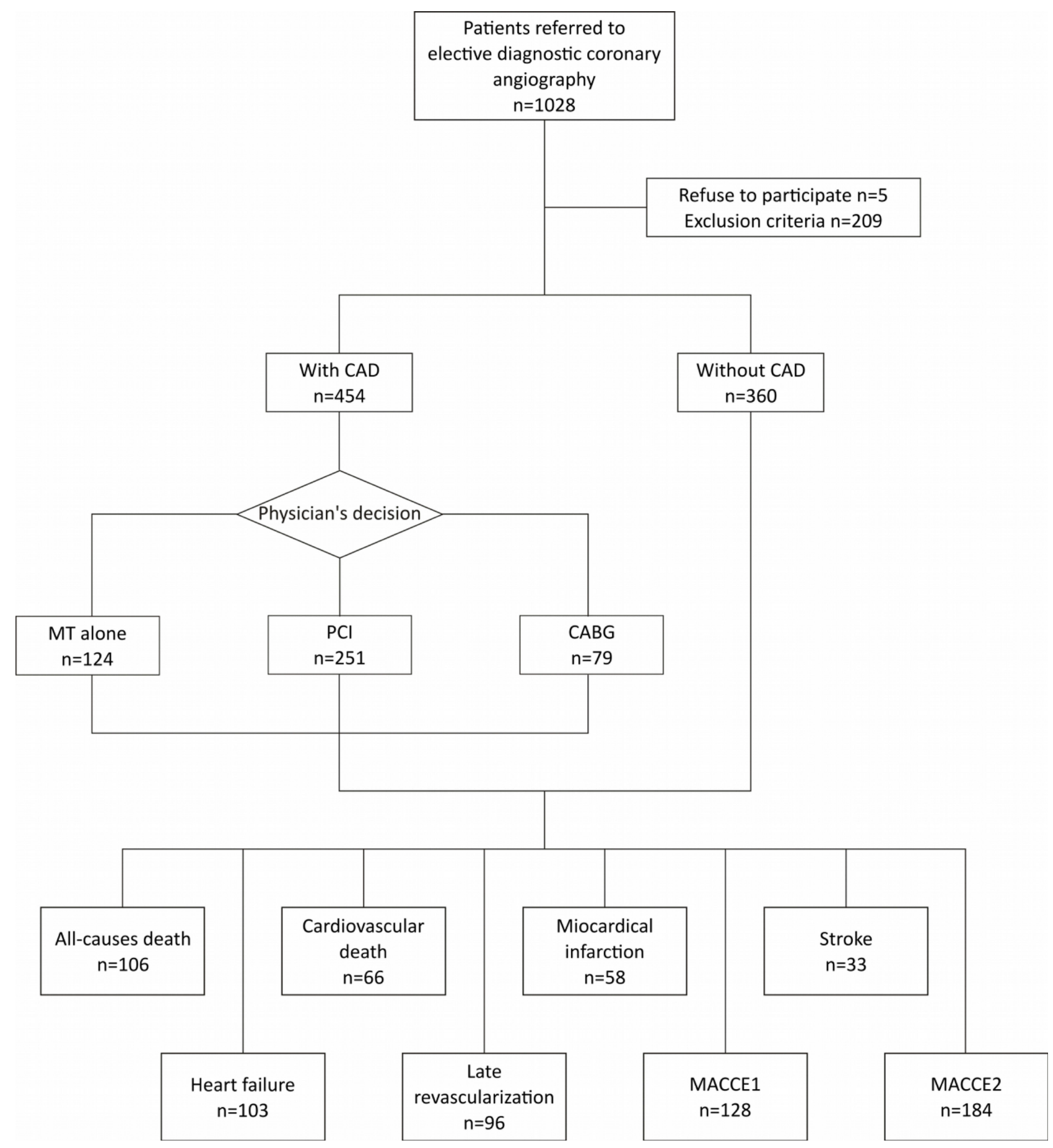

Figure I Study flowchart. PCI, percutaneous coronary intervention; CABG, coronary artery bypass grafting; CAD, coronary artery disease; MACCE, major adverse cardiac and cerebral events, as defined in Methods section.

failure and lower ejection fraction when compared to patients submitted to percutaneous revascularization.

Patients undergoing medical treatment only presented lower SXscores than the other groups. The CABG group presented significantly higher SXscores. When SXscore was stratified into categories, medical treatment only and PCI groups showed more patients in the low category and the CABG group showed a higher prevalence in intermediate and high categories.

\section{Incidence of Major Cardiovascular Events}

Table 2 shows the clinical outcomes in patients with and without CAD. All-cause death was significantly more frequent in patients subjected to medical therapy and to 
Table I Baseline Clinical and Angiographic Characteristics

\begin{tabular}{|c|c|c|c|c|c|}
\hline Baseline Characteristics ${ }^{\#}$ & MT Alone $(n=124)$ & $P C I(n=25 I)$ & CABG $(n=79)$ & Without CAD $(n=360)$ & $P$ value \\
\hline Age (years) & $61.9 \pm 10.2^{b}$ & $60.8 \pm 9.4^{\mathrm{ab}}$ & $61.7 \pm 8.4^{\mathrm{b}}$ & $58.1 \pm 10.3^{\mathrm{a}}$ & $<0.001$ \\
\hline Male & $69(55.6)$ & $173(68.9)^{*}$ & $54(68.4)^{*}$ & $165(45.8)^{* *}$ & $<0.001$ \\
\hline Race white & $87(70.2)$ & $176(70.1)$ & $6 \mathrm{I}(77.2)$ & $269(74.7)$ & 0.424 \\
\hline Years at school & $5(3-8)^{a b}$ & $5(4-9)^{\mathrm{b}}$ & $6(4-10)^{a b}$ & $5(3-8)^{a}$ & 0.001 \\
\hline BMI $(\mathrm{kg} / \mathrm{m} 2)$ & $28.9 \pm 5.2^{\mathrm{ab}}$ & $28.1 \pm 4.3^{\mathrm{ab}}$ & $27.4 \pm 4.2^{\mathrm{a}}$ & $29.3 \pm 5.6^{\mathrm{b}}$ & 0.002 \\
\hline $\mathrm{SBP}(\mathrm{mmHg})$ & $141.9 \pm 23.5^{\mathrm{ab}}$ & $|4| . \mid \pm 23.9^{\mathrm{ab}}$ & $144.8 \pm 20.6^{b}$ & $|37.9 \pm 21 .|^{\mathrm{a}}$ & 0.036 \\
\hline $\mathrm{DBP}(\mathrm{mmHg})$ & $79.7 \pm 11.9$ & $81.5 \pm 12.9$ & $83.2 \pm 11.6$ & $80.5 \pm 11.6$ & 0.154 \\
\hline Diabetes mellitus & $42(33.9)$ & 7I (28.3) & $31(39.2)^{*}$ & $81(22.5)^{* *}$ & 0.006 \\
\hline Hypertension & 114 (91.9) & $236(94)^{*}$ & $76(96.2)^{*}$ & $293(8 I .4)^{* *}$ & $<0.001$ \\
\hline Previous myocardial infarction & $45(36.3)^{* *}$ & $127(50.6)$ & $51(64.6)^{*}$ & - & $<0.001$ \\
\hline LVEF $(\%)$ & $62.6 \pm 14.1^{\mathrm{ab}}$ & $63.2 \pm 14.7^{b}$ & $58.3 \pm 15.9^{a}$ & $67.5 \pm 10.7^{c}$ & $<0.001$ \\
\hline $\mathrm{HF}$ & $19(15.3)$ & $40(15.9)$ & $17(2 \mid .5)^{*}$ & $27(7.5)^{* *}$ & 0.001 \\
\hline Glucose (mg/dL) & $103.4 \pm 33.1^{\mathrm{a}}$ & $106.5 \pm 27.8^{\mathrm{ab}}$ & $114.7 \pm 45.9^{\mathrm{b}}$ & $103.0 \pm 27.4^{\mathrm{a}}$ & 0.016 \\
\hline Total Cholesterol (mg/dL) & $170.4 \pm 47.3$ & $170.6 \pm 45.3$ & $176.6 \pm 51.8$ & $172.0 \pm 40.0$ & 0.734 \\
\hline HDL-C (mg/dL) & $4 \mathrm{I} .4 \pm 1 \mathrm{I} . \mathrm{I}^{\mathrm{ab}}$ & $39.7 \pm 9.9^{\mathrm{a}}$ & $40.7 \pm 10.1^{\mathrm{a}}$ & $44.5 \pm 11.4^{\mathrm{b}}$ & $<0.001$ \\
\hline Triglycerides (mg/dL) & $119.5(87.0-173.8)$ & $125(90.0-169.0)$ & $122(9|-| 76)$ & II $(8 I-158.0)$ & 0.238 \\
\hline Creatinine (mg/dL) & $0.69 \pm 0.21$ & $0.71 \pm 0.21$ & $0.72 \pm 0.22$ & $0.66 \pm 0.24$ & 0.105 \\
\hline hs-CRP (mg/dL) & $2.5(0.8-5.8)$ & $2.8(0.9-7.0)$ & $2.1(0.8-5.0)$ & $2.2(0.8-5.1)$ & 0.127 \\
\hline Smoking & $85(68.5)$ & $168(66.9)^{*}$ & $44(55.7)$ & $207(57.5)^{* *}$ & 0.026 \\
\hline Current smoking & $16(12.9)$ & $40(15.9)$ & $3(3.8)^{* *}$ & $5 I(14.2)$ & 0.050 \\
\hline Chest Pain & $29(23.4)$ & $44(17.5)^{* *}$ & $15.0(19)$ & $139(38.6)^{*}$ & $<0.001$ \\
\hline Dyspnea & $44(35.5)$ & $68(27.1)^{* *}$ & $26(32.9)$ & $167(46.4)^{*}$ & $<0.001$ \\
\hline 10-year ASCVD risk & $16.7(8.2-24.2)^{\mathrm{b}}$ & $15.6(9.1-23.4)^{b}$ & $19.6(11.2-28.1)^{b}$ & $10.9(5.8-11.7)^{\mathrm{a}}$ & $<0.001$ \\
\hline SXscore ${ }^{* * *}$ & $4.3(0-1 I)^{\mathrm{a}}$ & $8(5-13)^{b}$ & $21.5(13-26.5)^{c}$ & - & $<0.001$ \\
\hline Low SXscore & $117(94.4)^{*}$ & $235(93.6)^{*}$ & $49(62)^{* *}$ & - & $<0.001$ \\
\hline Intermediate SXscore & $5(4)^{* *}$ & $15(6)^{* *}$ & $21(28.6)^{*}$ & - & \\
\hline High SXscore & $2(1.6)$ & I $(0.4)^{* *}$ & $9(I I .4)^{*}$ & - & \\
\hline \multicolumn{6}{|l|}{ Indication of coronary angiography } \\
\hline Suggestive symptoms of CAD & $100(80.6)^{* *}$ & $209(83.3)^{* *}$ & $72(91.1)$ & $338(93.9)^{*}$ & $<0.001$ \\
\hline With a positive noninvasive test & $50(40.3)$ & $86(34.3)^{* *}$ & $38(48.1)$ & $160(44.4)$ & 0.043 \\
\hline Other complaints & II (8.9) & $16(6.4)$ & $8(10.1)$ & $32(8.9)$ & 0.616 \\
\hline
\end{tabular}

Notes: *Statistically significant positive association by adjusted residuals test to $5 \%$ of significance. **Statistically significant negative association by adjusted residuals test to

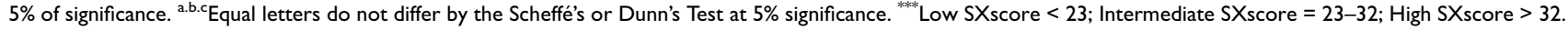
\#Variables were described by mean \pm SD, median (P25-P75) or as number (percentage).

Abbreviations: ASCVD, atherosclerotic cardiovascular disease; BMI, body mass index; CABG, coronary artery bypass grafting; CAD, coronary artery disease; HDL-C, high-density lipoprotein cholesterol; hs-CRP, high-sensitivity C-reactive protein; DBP, diastolic blood pressure; HF, heart failure; LVFE, left ventricular fraction ejection; MT, medical-therapy; PCI, percutaneous coronary intervention; SBP, systolic blood pressure; SXscore, SYNTAX score.

CABG. Cardiovascular death was higher in patients submitted to $\mathrm{CABG}$ than other groups. The incidence of MI and MACCE-2 was higher in patients subjected to myocardial revascularization procedures than in patients without CAD. Late revascularizations, surgical or percutaneous, were more frequent in patients initially submitted to PCI. Heart failure and MACCE-1 were more frequent in patients submitted to CBAG. Patients without CAD had, in general, a better prognosis than patients with CAD treated by any method.

Figure 2 shows the event-free survival curves for allcause death, cardiovascular death, MACCE-1 and
MACCE-2. All-cause death (Panel A), cardiovascular death (Panel B) and MACCE-1 (panel C) were not different by treatment strategies. Panel D shows that the incidence of MACCE-2 (with the inclusion of late revascularization) was significantly higher in participants submitted to PCI than in those submitted to clinical treatment.

The risks of death and major cardiovascular events according to the treatment group, considering medical therapy as reference are shown in Table 3. Deaths by any cause were less frequent in patients submitted to PCI, being marginally significant in the full model. CABG showed a qualitatively similar but not significant association with all-cause 
Table 2 Major Clinical Outcomes According to the Treatment of the Patients

\begin{tabular}{|c|c|c|c|c|c|}
\hline Type of Event ${ }^{\#}$ & MT Alone $(n=124)$ & $P C I(n=25 I)$ & CABG $(n=79)$ & Without CAD $(n=360)$ & $P$ value \\
\hline All-cause death & $24(19.4)^{*}$ & $25(10)$ & $16(20.3)^{*}$ & $4 I(11.4)$ & 0.012 \\
\hline Cardiovascular death & II (8.9) & $13(5.2)$ & $13(16.5)^{*}$ & $29(8.1)$ & 0.016 \\
\hline Myocardial infarction & $13(10.5)$ & $28(11.2)^{*}$ & $15(19)^{*}$ & $2(0.6)^{* *}$ & $<0.001$ \\
\hline Stroke & $4(3.2)$ & $7(2.8)$ & $6(7.6)$ & $16(4.4)$ & 0.268 \\
\hline Heart failure & $19(15.3)$ & $40(15.9)$ & $17(2 \mid .5)^{*}$ & $27(7.5)^{* *}$ & 0.001 \\
\hline Late Revascularization & $18(14.5)$ & $66(26.3)^{*}$ & $9(11.4)$ & $3(0.8)^{* *}$ & $<0.001$ \\
\hline MACCE-I & $24(19.4)$ & $39(15.5)$ & $24(30.4)^{*}$ & $41(11.4)^{* *}$ & $<0.001$ \\
\hline MACCE-2 & $30(24.2)$ & $82(32.7)^{*}$ & $30(38)^{*}$ & $42(11.7)^{* *}$ & $<0.001$ \\
\hline
\end{tabular}

Notes: *Statistically significant positive association by adjusted residuals test to $5 \%$ of significance. **Statistically significant negative association by adjusted residuals test to $5 \%$ of significance. "Variables were described as number (percentage).

Abbreviations: MACCE, major adverse cardiac and cerebral events; MACCE-I, composite of cardiovascular death, myocardial infarction and stroke; MACCE 2, all components of MACCE-I plus late revascularization; MT, medical-therapy.

deaths in the full model. PCI was also associated with a lower incidence of cardiovascular (CV) deaths, while the estimates for $\mathrm{CABG}$ were all in the risk side in the three models, but the estimates were not statistically significant. The associations with myocardial infarction, stroke and heart failure were not significant for $\mathrm{PCI}$ and $\mathrm{CABG}$, while late revascularization was significantly higher in the PCI group. PCI had a trend towards benefit for the combined incidence of cardiovascular outcomes (MACCE-1), but showed a higher incidence of these events when taking into account late revascularization. Surgery did not protect against the incidence of major cardiovascular events combined (MACCE-1) or associated with late revascularization (MACCE-2).

The risks for MACCE-2 were significantly higher for patients treated by PCI than CABG in patients with diabetes (but both significantly higher than in patients treated medically) (Figure 3). There was no significant interaction with left ventricular ejection fraction (LVEF), SBP and previous MI. For other outcomes (all-cause death, cardiovascular death, myocardial infarction, stroke, heart failure and MACCE-1), there was no interaction between those conditions and the association with PCI and CABG.

\section{Discussion}

This real-world cohort study investigated clinical outcomes of patients submitted to diagnostic coronary angiography and referred to medical therapy, PCI or CABG. All comers were meticulously evaluated at baseline and followed on average for $6 \pm 1.9$ years. After adjusting for potential confounding variables, patients submitted to PCI had a lower all-cause mortality rate than patients treated medically. A non-significant trend for lower cardiovascular mortality was observed in patients treated by PCI. The benefit in the prevention of all-cause mortality occurred at the expense of repeated revascularization procedures. Patients treated surgically had more severe disease and comorbid conditions than patients treated medically or percutaneously. Even after adjusting for baseline characteristics, patients submitted to CABG did not have a better prognosis than patients treated medically. On the contrary, patients treated surgically had higher cardiovascular mortality combined with the incidence of major cardiovascular events. These results were not influenced by the presence of diabetes mellitus, blood pressure levels, left ventricular ejection fraction and previous MI. The incidence of nonfatal cardiovascular events was not associated with either revascularization modality. Patients without $\mathrm{CAD}$ had an overall better prognosis than their counterparts with $\mathrm{CAD}$, despite more frequent complaints of dyspnea and chest pain at the baseline evaluation.

The higher risk for cardiovascular mortality associated with major non-fatal $\mathrm{CV}$ events in patients submitted to CABG may have resulted from residual confounding, ie, the absence of control for confounding variables not available in the database. Nonetheless, most clinical and angiographic differences between patients treated medically or surgically were included in the models. Strictly, it is unlikely that the worse outcomes with surgery would turn to protection with the adjustment of unknown confounders. The interpretation that $\mathrm{CABG}$ was not able to diminish the worse baseline prognosis of patients in comparison with those treated medically is valid. In fact, our findings are in accordance with the results of the classical Coronary Artery Surgery Studies (CASS), ${ }^{27}$ Veterans Administration Cooperative Study $^{28}$ and European Coronary Surgery Study, ${ }^{29}$ which did not demonstrate the superiority of surgery over medical treatment in the main cohorts. The beneficial effects in patients with multiarterial disease (or left main 


\section{A All-cause death free-time}

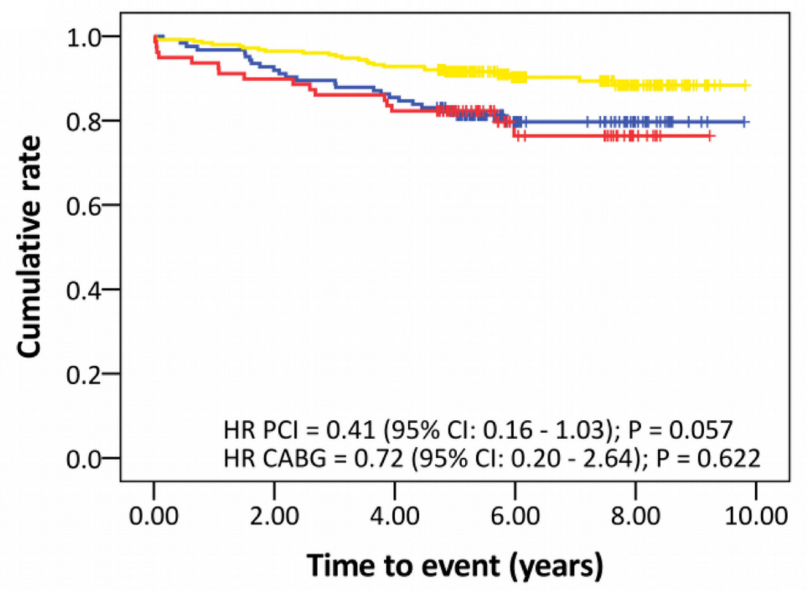

\section{MACCE 1 free-time}

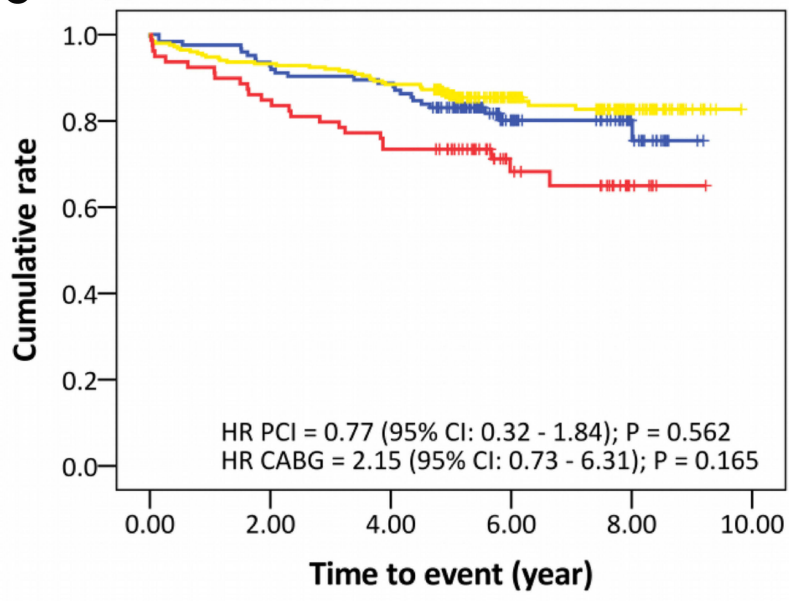

B Cardiovascular death free-time

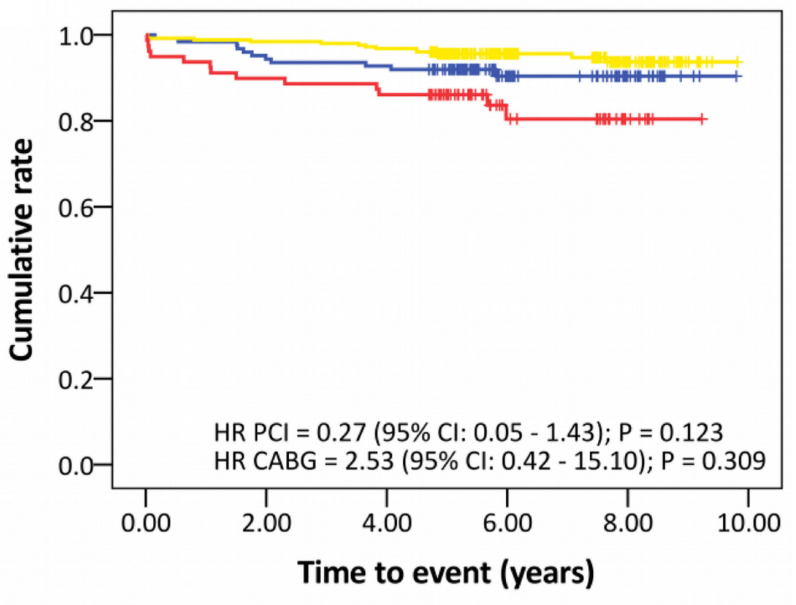

D MACCE 2 free-time

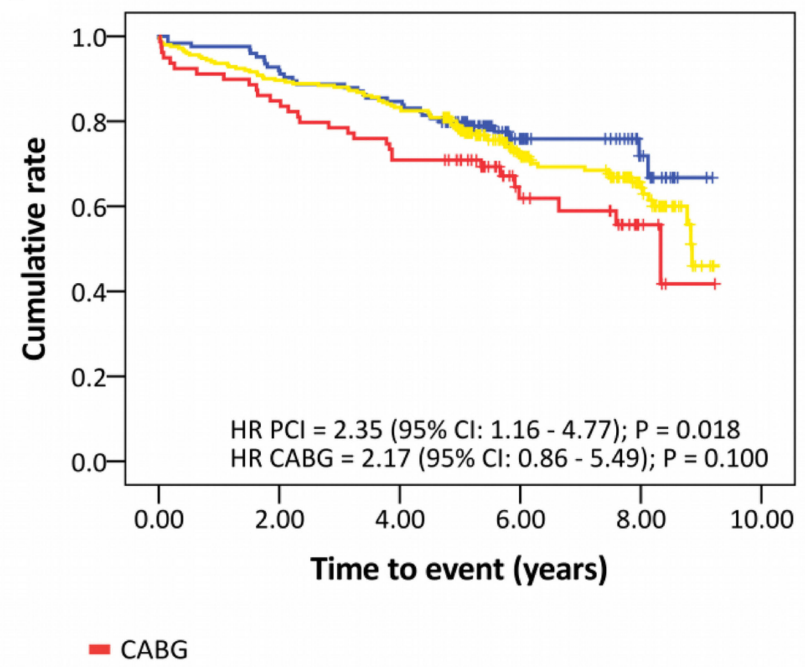

Figure 2 Event-free survival curves for all-cause death, cardiovascular death, MACCE-I, and MACCE-2 adjusted for age, sex, school degree, BMI, DM, hypertension, chest pain, HDL-C, creatinine, hs-CRP, smoking, HF, LVEF, MI before the index procedure and SXscore. CABG, coronary-artery bypass grafting; MT, medical-therapy; PCl, percutaneous coronary intervention. Abbreviations as in Table I.

disease), particularly in patients with heart failure, could not be explored in our cohort, because of the small sample and relatively good health status of our patients.

Overall, our findings are in agreement with MASS II, ${ }^{14}$ COURAGE $^{15}$ and the BARI-2D ${ }^{16}$ trials. In BARI-2D, a strategy of revascularization associated with medical-therapy was not better than medical-therapy alone to reduce death or major cardiovascular events. Similarly, in the COURAGE trial, PCI did not reduce the risk of death or MI, even in patients with diabetes. Furthermore, the incidence of long-term events with medical-therapy was similar to that of PCI, as was also observed in 5 and 10-year follow-up results of the MASS II trial. Also, many studies did not show that the combination of percutaneous and medical treatment reduces major cardiovascular endpoints compared to the medical treatment alone. ${ }^{15,30,31}$ Even when adjusted for possible confounders, the risk of late revascularization remains high for patients submitted to PCI compared to medical treatment, meaning that PCI is an important risk factor for this outcome. ${ }^{32-34}$ Recently, analyses of patients with baseline ischemia were published, with no advantage of percutaneous treatment over medical treatment. ${ }^{17,18}$ Subgroup analysis in patients with ischemia from the COURAGE trial ${ }^{18}$ and a meta-analysis with four additional studies ${ }^{18}$ demonstrated that revascularization was ineffective in preventing events in patients with asymptomatic or mildly symptomatic CAD. The ongoing ISCHEMIA clinical trial ${ }^{35}$ intends to answer this 
Table 3 Cox Regression Analysis to Evaluate the Effect of the Treatment on the Outcomes After Diagnostic Coronary Angiography with Adjustment for Confounding Factors

\begin{tabular}{|c|c|c|c|c|c|}
\hline \multirow[t]{2}{*}{ Outcome } & \multirow[t]{2}{*}{ Models $^{\mathrm{a}}$} & \multicolumn{2}{|l|}{$\mathbf{P C l}^{*}$} & \multicolumn{2}{|l|}{ CABG* } \\
\hline & & HR (95\% IC) & $P$ value & HR (95\% Cl) & $P$ value \\
\hline \multirow[t]{3}{*}{ All-cause death } & I & $0.49(0.28-0.87)$ & 0.014 & $1.15(0.6 \mathrm{I}-2.17)$ & 0.676 \\
\hline & 2 & $0.41(0.16-1.05)$ & 0.062 & $0.88(0.28-2.74)$ & 0.828 \\
\hline & 3 & $0.41(0.16-1.03)$ & 0.057 & $0.72(0.20-2.64)$ & 0.622 \\
\hline \multirow[t]{3}{*}{ Cardiovascular death } & 1 & $0.61(0.27-1.36)$ & 0.226 & $2.21(0.98-4.98)$ & 0.056 \\
\hline & 2 & $0.26(0.05-I .4 I)$ & 0.120 & $2.28(0.49-10.59)$ & 0.293 \\
\hline & 3 & $0.27(0.05-1.43)$ & 0.123 & $2.53(0.42-15.10)$ & 0.309 \\
\hline \multirow[t]{3}{*}{ Myocardial infarction } & 1 & $1.10(0.57-2.14)$ & 0.772 & $1.94(0.92-4.10)$ & 0.082 \\
\hline & 2 & $1.12(0.38-3.29)$ & 0.835 & $2.07(0.66-6.47)$ & 0.211 \\
\hline & 3 & $1.12(0.38-3.29)$ & 0.839 & $1.66(0.45-6.10)$ & 0.447 \\
\hline \multirow[t]{3}{*}{ Stroke } & I & $0.91(0.26-3.12)$ & 0.879 & $2.53(0.7 \mid-9.03)$ & 0.154 \\
\hline & 2 & $0.67(0.06-7.18)$ & 0.670 & $8.67(0.91-82.98)$ & 0.061 \\
\hline & 3 & $0.69(0.06-7.54)$ & 0.760 & $9.97(0.57-174.58)$ & 0.115 \\
\hline \multirow[t]{3}{*}{ Heart failure } & I & $1.10(0.63-1.92)$ & 0.740 & I.7I (0.87-3.34) & 0.119 \\
\hline & 2 & $1.31(0.52-3.28)$ & 0.566 & $1.99(0.78-5.08)$ & 0.152 \\
\hline & 3 & $1.34(0.53-3.39)$ & 0.542 & $1.66(0.61-4.53)$ & 0.328 \\
\hline \multirow[t]{3}{*}{ Late revascularization } & 1 & $1.78(1.05-3.03)$ & 0.034 & $0.85(0.38-1.90)$ & 0.689 \\
\hline & 2 & $4.15(1.67-10.29)$ & 0.002 & $2.08(0.63-6.88)$ & 0.230 \\
\hline & 3 & $3.63(1.46-9.04)$ & 0.006 & $1.24(0.34-4.55)$ & 0.750 \\
\hline \multirow[t]{3}{*}{ MACCE-I } & 1 & $0.82(0.49-1.37)$ & 0.448 & $1.78(1.01-3.14)$ & 0.048 \\
\hline & 2 & $0.80(0.34-1.89)$ & 0.603 & $2.57(1.05-6.30)$ & 0.039 \\
\hline & 3 & $0.77(0.32-1.84)$ & 0.562 & $2.15(0.73-6.31)$ & 0.165 \\
\hline \multirow[t]{3}{*}{ MACCE-2 } & 1 & $1.31(0.86-1.99)$ & 0.216 & $1.82(1.09-3.02)$ & 0.021 \\
\hline & 2 & $2.56(1.27-5.17)$ & 0.009 & $3.28(I .45-7.4 I)$ & 0.004 \\
\hline & 3 & $2.35(1.16-4.77)$ & 0.018 & $2.17(0.86-5.49)$ & 0.100 \\
\hline
\end{tabular}

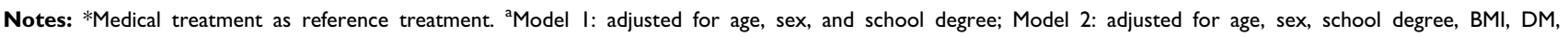
hypertension, chest pain, HDL-C, creatinine, hs-CRP, smoking, HF, LV ejection fraction and MI before the index procedure; and Model 3: adjusted for age, sex, school degree, BMI, DM, hypertension, chest pain, HDL-C, creatinine, hs-CRP, smoking, HF, LVEF, MI before the index procedure and SXscore.

Abbreviations: MACCE, major adverse cardiac and cerebral events; MACCE-I, composite of cardiovascular death, myocardial infarction and stroke; MACCE-2, outcomes of MACCE-I plus late revascularization. Abbreviations as in Table I.

question definitively. The first randomized trial with a control sham procedure failed to demonstrate the effectiveness of PCI to control symptoms and to increase exercise time. ${ }^{36}$

There are many clinical trials comparing the effectiveness of PCI and CABG. ${ }^{12,13,16,24,25,37-49}$ Our study was not primarily designed to investigate this question and lacks statistical power for direct comparisons. The trend for beneficial effects of PCI over all-cause and cardiovascular mortality, in comparison with no beneficial effects of surgery, was unexpected. Although the higher probability of death of patients subjected to surgical treatment, there was no significant difference among the groups during the follow-up period, similarly to the results of the 5-year follow-up of the SYNTAX trial ${ }^{40}$ and of a meta-analysis of randomized trials, ${ }^{12}$ except for cardiovascular death. Results of the 5-year follow-up of the SYNTAX trial ${ }^{40}$ and the NOBLE trial ${ }^{13}$ showed that surgical treatment presented significantly less MACCE than percutaneous treatment, in patients with more complex coronary artery disease, similar to the present study. Also, in meta-analyses of observational studies ${ }^{24}$ and randomized clinical trials, ${ }^{38,39,45}$ comparing surgical to percutaneous treatment, long-term mortality and clinical outcomes were similar for patients with multivessel CAD, except for late revascularization. The present cohort showed that, in the real world, percutaneous treatment is associated with a four-fold increased risk of repeat revascularization when compared 


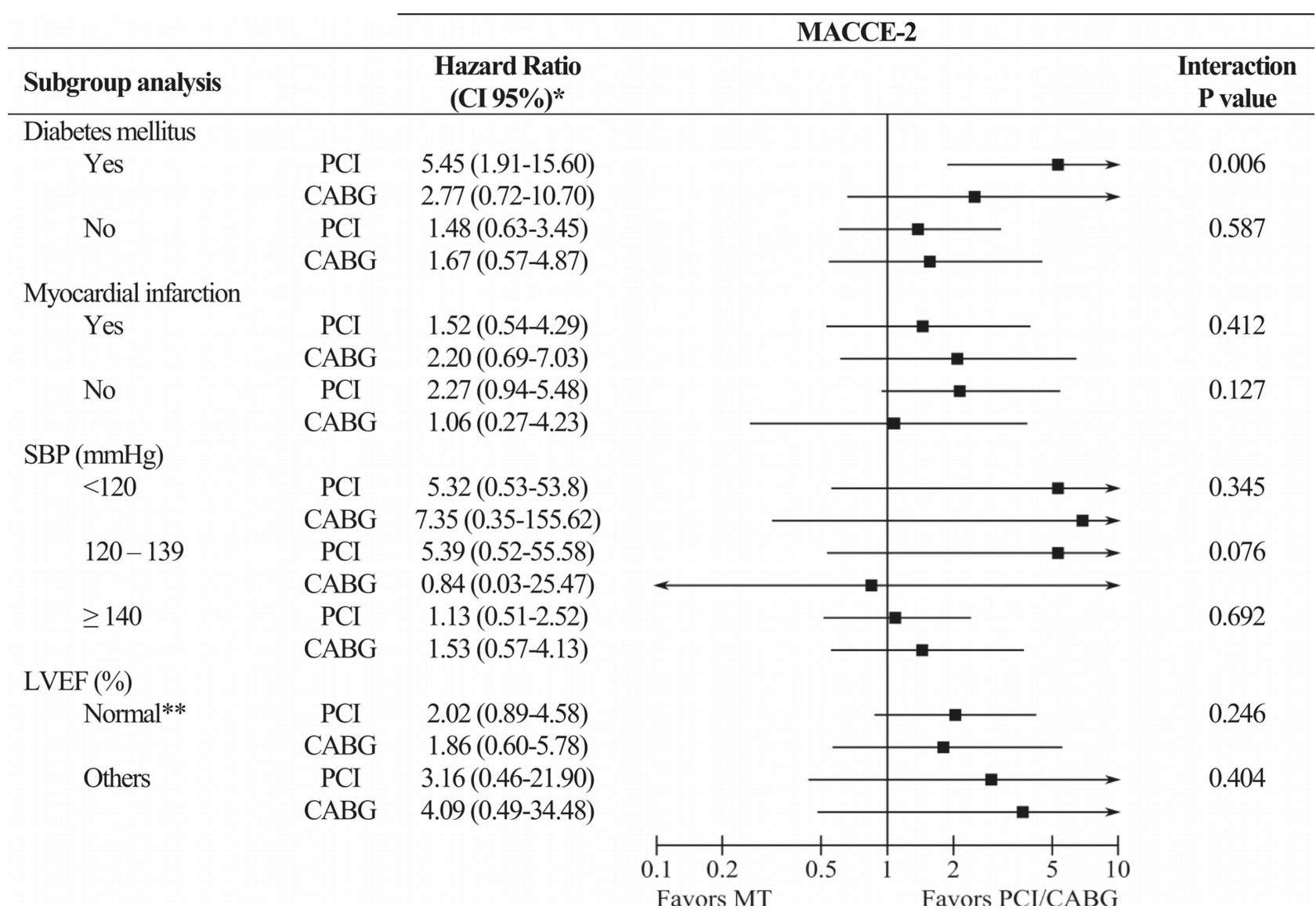

Figure 3 Hazard ratio for MACCE-2 in participants stratified by diabetes mellitus, myocardial infarction, categories of systolic blood pressure, and left ventricular ejection fraction. *Adjusted for model 3 as. Table 3. **Normal for male $\geq 52 \%$ and for female $\geq 54 \%$. Abbreviations as in Table 1 .

to medical therapy alone. Similar results were seen in a meta-analysis of observational studies of 24,268 patients with multivessel CAD treated with surgery or drug-eluting stents, in which the frequency of repeat revascularization was also roughly 4-fold higher after DES implantation. ${ }^{24}$ The possibility of residual confounding cannot be fully discarded in our study, and the disease severity can be the underlying reason for the indirect superiority of PCI over CABG. Patients submitted to surgical revascularization had more extensive CAD, as expressed by the higher SYNTAX scores of this group.

Our study has limitations that deserve mention. The nonrandomized design precludes to fully controlling for confounding factors. Even with adjustment for most risk factors and severity of coronary disease, the possibility of residual confounding cannot be ruled out. On the other hand, the proposal of the study was to assess the beneficial effects of treatments in the real world. Our sample size of patients submitted to $\mathrm{CABG}$ was small. In the face of the higher incidence of combined cardiovascular morbidity and death in patients submitted to surgery, it is unlikely that this risk would be reversed in a study with a larger sample size. And finally, our study was performed at a single center.

\section{Conclusions}

Patients with newly diagnosed CAD by elective coronary angiography did not have a better prognosis when submitted to CABG comparatively to medical treatment. Patients treated with PCI had a trend for the lower incidence of all-cause death and lower risk of MACCE-2, but at the expense of additional revascularization procedures. Patients without significant CAD had a similar prognosis than CAD patients treated with medical therapy. Unless new findings from randomized clinical trials show the superiority of surgical or percutaneous therapies, patients with chronic, stable, coronary artery disease should be preferentially managed with medical therapy.

\section{Abbreviations}

ASCVD, atherosclerotic cardiovascular disease; BMI, body mass index; CABG, coronary artery bypass grafting; 
CAD, coronary artery disease; CHD, coronary heart disease; $\mathrm{CV}$, cardiovascular; DBP, diastolic blood pressure; DES, drug-eluting stents; DM, diabetes mellitus; ECG, electrocardiogram; HDL-C, high-density lipoprotein cholesterol; HF, heart failure; hs-CRP, high-sensitivity C-reactive protein; LVEF, left ventricular ejection fraction; MACCE, major adverse cardiac or cerebral events; MI, myocardial infarction; MT, medical-therapy; PCI, percutaneous coronary intervention; SBP, systolic blood pressure; SXscore, SYNTAX score; SYNTAX, SYNergy between percutaneous coronary intervention with TAXus ${ }^{\circledR}$ and Cardiac Surgery Study.

\section{Data Sharing Statement}

The data that support the findings of this study are available on request from the corresponding author ASA. The data are not publicly available due to containing information that could compromise research participant privacy/ consent.

\section{Ethics Approval and Informed Consent}

The study protocol was approved by the Ethics Committee of the Hospital de Clínicas de Porto Alegre, registered under no. 13-0171, which is accredited by the Office for Human Research Protections as an Institutional Review Board. This study was conducted in accordance with the Declaration of Helsinki. All participants provided informed consent.

\section{Consent for Publication}

Informed consent has been obtained from all participants included in the analyzed studies.

\section{Author Contributions}

All authors contributed to data analysis, drafting or revising the article, gave final approval of the version to be published, and agree to be accountable for all aspects of the work.

\section{Funding}

Financial support was provided by Research Support Fund at Hospital de Clínicas de Porto Alegre (FIPE-HCPA), registered under no. 13-0171, and did not receive any other specific grant from funding agencies in the public, commercial, or not-for-profit sectors.

\section{Disclosure}

The authors declare that they have no competing interests.

\section{References}

1. Fihn SD, Blankenship JC, Alexander KP, et al. 2014 ACC/AHA/ AATS/PCNA/SCAI/STS focused update of the guideline for the diagnosis and management of patients with stable ischemic heart disease: a report of the American college of cardiology/American heart association. Task force on practice guidelines, and the American association for thoracic surgery, preventive cardiovascular nurses association, society for cardiovascular angiography and interventions, and society of thoracic surgeons. Circulation. 2014;130(19):17491767. doi:10.1161/CIR.0000000000000095

2. Trial Participants RITA. Coronary angioplasty versus coronary artery bypass surgery: the Randomized Intervention Treatment of Angina (RITA) trial. Lancet. 1993;341(8845):573-580. doi:10.1016/01406736(93)90348-K

3. Rodriguez A, Boullon F, Perez-Baliño N, et al. Argentine randomized trial of percutaneous transluminal coronary angioplasty versus coronary artery bypass surgery in multivessel disease (ERACI): inhospital results and 1-year follow-up. J Am Coll Cardiol. 1993;22 (4):1060-1067. doi:10.1016/0735-1097(93)90416-X

4. Trial Participants CABRI. First-year results of CABRI (coronary angioplasty versus bypass revascularization investigation). Lancet. 1995;346(8984):1179-1184

5. King SB, Lembo NJ, Weintraub WS, et al. A randomized trial comparing the coronary angioplasty with coronary bypass surgery. $N$ Engl $J$ Med. 1994;331(16):1044-1050. doi:10.1056/NEJM199410203311602

6. Hamm CW, Reimers J, Ischinger T, Rupprecht HJ, Berger J, Bleifeld W. For the German angioplasty bypass surgery investigation. A randomized study of coronary angioplasty compared with bypass surgery in patients with symptomatic multivessel coronary disease. $N$ Engl J Med. 1994;331(16):1037-1043. doi:10.1056/NEJM 199410203311601

7. The Bypass Angioplasty Revascularization Investigation (BARI) Investigators. Comparison of coronary bypass surgery with angioplasty in patients with multivessel disease. $N$ Engl J Med. 1996;335 (4):217-225. doi:10.1056/NEJM199607253350401

8. Zhang Z, Mahoney EM, Stables RH, et al. Disease-specific health status after stent-assisted percutaneous coronary intervention and coronary artery bypass surgery: one-year results from the Stent or Surgery trial. Circulation. 2003;108(14):1694-1700. doi:10.1161/01. CIR.0000087600.83707.FD

9. Rodriguez A, Bernardi V, Navia J, et al. Argentine randomized study: coronary angioplasty with stenting versus coronary bypass surgery in patients with multiple-vessel disease (ERACI II): 30 day and oneyear follow-up results. $J$ Am Coll Cardiol. 2001;37(1):51-58. doi:10.1016/S0735-1097(00)01052-4

10. Serruys PW, Unger F, Sousa JE, et al. Comparison of coronary-artery bypass surgery and stenting for the treatment of multivessel disease. $N$ Engl J Med. 2001;344(15):1117-1124. doi:10.1056/NEJM200 104123441502

11. Head SJ, Davierwala PM, Serruys PW, et al. Coronary artery by-pass grafting vs. percutaneous coronary intervention for patients with three-vessel disease: final five-year follow-up of the SYNTAX trail. Eur Heart J. 2014;35(40):2821-2830. doi:10.1093/eurheartj/ehu213

12. Sipahi I, Akay MH, Dagdelen S, Blitz A, Alhan C. Coronary artery bypass grafting vs percutaneous coronary intervention and long-term mortality and morbidity in multivessel disease meta-analysis of randomized clinical trials of the arterial grafting and stenting era. JAMA Intern Med. 2014;174(2):223-230. doi:10.1001/jamainternmed.2013. 12844.

13. Mäkikallio T, Holm NR, Lindsay M, et al. Percutaneous coronary angioplasty versus coronary artery bypass grafting in treatment of unprotected left main stenosis (NOBLE): a prospective, randomized, open-label, non-inferiority trial. Lancet. 2016;388(10061):27432752. doi:10.1016/S0140-6736(16)32052-9 
14. Hueb W, Lopes N, Gersh BJ, et al. Ten-year follow-up survival of the medicine, angioplasty, or surgery study (MASS II). A randomized controlled clinical trial of 3 therapeutic strategies for multivessel coronary artery disease. Circulation. 2010;122(10):949-957. doi:10. 1161/CIRCULATIONAHA.109.911669

15. Boden WE, O'Rourke RA, Teo KK. et al; for the COURAGE trial research group. Optimal medical therapy with or without PCI for stable coronary disease. $N$ Engl J Med. 2007;356(15):1503-1516. doi:10.1056/NEJMoa070829

16. The BARI 2D Study Group. A randomized trial of therapies for type 2 diabetes and coronary artery disease. $N$ Engl J Med. 2009;360 (24):2503-2515. doi:10.1056/NEJMoa0805796

17. Shaw LJ, Weintraub WS, Maron DJ, et al. Baseline stress myocardial perfusion imaging results and outcomes in patients with stable ischemic heart disease randomized to optimal medical therapy with or without percutaneous coronary intervention. Am Heart J. 2012;164 (2):243-250. doi:10.1016/j.ahj.2012.05.018

18. Stergiopoulos K, Boden WE, Hartigan P, et al. Percutaneous coronary intervention outcomes in patients with stable obstructive coronary artery disease and myocardial ischemia. A collaborative meta-analysis of contemporary randomized clinical trials. JAMA Intern Med. 2014;174(2):232-240. doi:10.1001/jamainternmed.2013. 12855

19. Myers WO, Gersh BJ, Fisher LD, et al. Medical versus early surgical therapy in patients with triple-vessel disease and mild angina pectoris: a CASS registry study of survival. Ann Thorac Surg. 1987;44 (5):471-486. doi:10.1016/S0003-4975(10)62104-2

20. Muhlbaier LH, Pryor DB, Rankin JS, et al. Observational comparison of event-free survival with medical and surgical therapy in patients with coronary artery disease 20 Years of Follow-Up. Circulation. 1992;86(Suppl 5):II198-204.

21. Caracciolo EA, Davis KB, Sopko G, et al. Comparison of surgical and medical group survival in patients with left main equivalent coronary artery disease. Long-term CASS experience. Circulation. 1995;91(9):2335-2344. doi:10.1161/01.CIR.91.9.2335

22. Myers WO, Blackstone EH, Davis K, Foster ED, Kaiser GC, Registry CASS. Long Term Surgical Survival. J Am Coll Cardiol. 1999;33(2):488-498. doi:10.1016/S0735-1097(98)00563-4

23. Hannan EL, Wu C, Walford G, et al. Drug-eluting stents vs. coronary-artery bypass grafting in multivessel coronary disease. $N$ Engl $J$ Med. 2008;358(4):331-341. doi:10.1056/NEJMoa071804

24. Benedetto U, Melina G, Angeloni E, et al. Coronary artery bypass grafting versus drug-eluting stents in multivessel coronary disease. A meta-analysis on 24,268 patients. Eur J Cardiothorac Surg. 2009;36 (4):611-615. doi:10.1016/j.ejcts.2009.03.012

25. Serruys PW, Morice MC, Kappetein AP. et al; for the SYNTAX investigators. Percutaneous coronary intervention versus coronary-artery bypass grafting for severe coronary artery disease. $N$ Engl J Med. 2009;360(10):961-972. doi:10.1056/NEJMoa0804 626

26. The ARIC lnvestigators. The Atherosclerosis Risk in Communities (ARIC) Study: design and Objectives. Am J Epidemiol. 1989;129 (4):687-702. doi:10.1093/oxfordjournals.aje.a115184

27. Principal Investigators CASS, Associates T. Coronary Artery Surgery Study (CASS): a randomized trial of coronary artery bypass surgery. Survival data. Circulation. 1983;68:939-950.

28. The Veterans Administration Coronary Artery Bypass Surgery Cooperative Study Group. Eleven-year survival in the Veterans Administration randomized trial of coronary bypass surgery for stable angina. $N$ Engl J Med. 1984;311(21):1333-1339. doi:10.1056/NEJM 198411223112102

29. Varnauskas E. The European coronary surgery study group. Twelveyear follow-up of survival in the randomized european coronary surgery study. $N$ Engl J Med. 1988;319(6):332-337. doi:10.1056/ NEJM198808113190603
30. Henderson RA, Pocock SJ, Clayton TC. et al; for the second randomized intervention treatment of angina (RITA-2) trial participants. Seven-year outcome in the RITA-2 trial: coronary angioplasty versus medical therapy. $J$ Am Coll Cardiol. 2003;42(7):1161-1170. doi:10.1016/S0735-1097(03)00951-3

31. Chang M, Lee CW, Ahn JM, et al. Outcomes of Coronary artery bypass graft surgery versus drug-eluting stents in older adults. $J \mathrm{Am}$ Geriatr Soc. 2017;65(3):625-630. doi:10.1111/jgs.14780

32. Chieffo A, Magni V, Latib A, et al. 5-Year outcomes following percutaneous coronary intervention with drug-eluting stent implantation versus coronary artery bypass graft for unprotected left main coronary artery lesions. The milan experience. JACC Cardiovasc Interv. 2010;3(6):595-601. doi:10.1016/j.jcin.2010.03.014

33. Morice MC, Serruys PW, Kappetein AP, et al. Five-year outcomes in patients with left main disease treated with either percutaneous coronary intervention or coronary artery bypass grafting in the synergy between percutaneous coronary intervention with taxus and cardiac surgery trial. Circulation. 2014;129(23):2388-2394. doi:10.1161/ CIRCULATIONAHA.113.006689

34. Lim JY, Deo SV, Kim WS, Altarabsheh SE, Erwin PJ, Park SJ. Drugeluting stents versus coronary artery bypass grafting in diabetic patients with multivessel disease: a meta-analysis. Heart Lung Circ. 2014;23(8):717-725. doi:10.1016/j.hlc.2014.02.005

35. Hochman J, Maron D, Boden W, et al. International Study of Comparative Health Effectiveness With Medical and Invasive Approaches (ISCHEMIA). Available from: https://www.ischemia trial.org/. Accessed June 30, 2020. Accessed July 30, 2017.

36. Al-Lamee R, Thompson D, Dehbi HM. et al; on behalf of the ORBITA investigators. Percutaneous coronary intervention in stable angina (ORBITA): a double-blind, randomised controlled trial. Lancet. 2018;391(10115):31-40. doi:10.1016/S0140-6736(17)32714-9

37. Hlatky MA, Boothroyd DB, Bravata DM, et al. Coronary artery bypass surgery compared with percutaneous coronary interventions for multivessel disease: a collaborative analysis of individual patient data from ten randomized trials. Lancet. 2009;373(9670):1190-1197. doi:10.1016/S0140-6736(09)60552-3

38. Athappan G, Patvardhan E, Tuzcu ME, Ellis S, Whitlow P, Kapadia SR. Left main coronary artery stenosis: a meta-analysis of drug-eluting stents versus coronary artery bypass grafting. JACC Cardiovasc Interv. 2013;6(12):1219-1230. doi:10.1016/j.jcin.2013.07.008

39. Sá MP, Soares AM, Lustosa PC, et al. Meta-analysis of 5674 patients treated with percutaneous coronary intervention and drug-eluting stents or coronary artery bypass graft surgery for unprotected left main coronary artery stenosis. Eur J Cardiothorac Surg. 2013;43 (1):73-80. doi:10.1093/ejcts/ezs204

40. Mohr FW, Morice MC, Kappetein AP, et al. Coronary artery bypass graft surgery versus percutaneous coronary intervention in patients with three-vessel disease and left main coronary disease: 5-year follow-up of the randomized, clinical SYNTAX trial. Lancet. 2013;381(9867):629-638. doi:10.1016/S0140-6736(13)60141-5

41. Park SJ, Ahn JM, Kim YH, et al. For the BEST trial investigators. Trial of everolimus-eluting stents or bypass surgery for coronary disease. $N$ Engl J Med. 2015;372(13):1204-1212. doi:10.1056/NEJMoa1415447

42. Park SJ, Kim YH, Park DW, et al. Randomized trial of stents versus bypass surgery for left main coronary artery disease. $N$ Engl $J$ Med. 2011;364(364):1718-1727. doi:10.1056/NEJMoa1100452

43. Ahn JM, Roh JH, Kim YH, et al. Randomized trial of stents versus bypass surgery for left main coronary artery disease: 5 -year outcomes of the PRECOMBAT study. J Am Coll Cardiol. 2015;65(20):21982206. doi:10.1016/j.jacc.2015.03.033

44. Lee CW, Ahn JM, Cavalcante R, et al. Coronary artery bypass surgery versus drug-eluting stent implantation for left main or multivessel coronary artery disease. A meta-analysis of individual patient data. JACC Cardiovasc Interv. 2016;9(24):2481-2489. doi:10.1016/j. jcin.2016.10.008 
45. Huang F, Lai W, Chan C, et al. Comparison of bypass surgery and drugeluting stenting in diabetic patients with left main and/or multivessel disease: a systematic review and meta-analysis of randomized and nonrandomized studies. Cardiol J. 2015;22(2):123-134. doi:10.5603/CJ. a2014.0036

46. Mack MJ, Head SJ, Holmes DR Jr, et al. Analysis of stroke occurring in the SYNTAX trial comparing coronary artery bypass surgery and percutaneous coronary intervention in the treatment of complex coronary artery disease. JACC Cardiovasc Interv. 2013;6(4):344-354. doi:10.1016/j.jcin.2012.11.010

47. Aronson D, Edelman ER. Revascularization for coronary artery disease in diabetes mellitus: angioplasty, stents and coronary artery bypass grafting. Rev Endocr Metab Disord. 2010;11(1):75-86. doi:10.1007/s11154-010-9135-3
48. Mack MJ, Banning AP, Serruys PW, et al. Bypass versus drug eluting stents at three years in SYNTAX patients with diabetes mellitus or metabolic syndrome. Ann Thorac Surg. 2011;92(6):2140-2146. doi:10.1016/j.athoracsur.2011.06.028

49. Farkouh ME, Domanski M, Sleeper LA. et al; for the FREEDOM trial investigators. Strategies for multivessel revascularization in patients with diabetes. $N$ Engl J Med. 2012;367(25):2375-2384. doi:10.1056/NEJMoa1211585

\section{Publish your work in this journal}

Vascular Health and Risk Management is an international, peerreviewed journal of therapeutics and risk management, focusing on concise rapid reporting of clinical studies on the processes involved in the maintenance of vascular health; the monitoring, prevention and treatment of vascular disease and its sequelae; and the involvement of metabolic disorders, particularly diabetes. This journal is indexed on PubMed Central and MedLine. The manuscript management system is completely online and includes a very quick and fair peerreview system, which is all easy to use. Visit http://www.dovepress. com/testimonials.php to read real quotes from published authors. 